\title{
Primary tumor inflammation in gross tumor volume as a prognostic factor for nasopharyngeal carcinoma patients
}

\author{
Hao Peng ${ }^{1}$, Lei Chen ${ }^{1}$, Ling-Long Tang ${ }^{1}$, Yuan Zhang ${ }^{1}$, Wen-Fei Li ${ }^{1}$, Yan-Ping Mao ${ }^{1}$, \\ Fan Zhang ${ }^{1}$, Rui Guo ${ }^{1}$, Li-Zhi Liu ${ }^{2}$, Li Tian'르, Ai-Hua Lin ${ }^{3}$, Ying Sun ${ }^{1}$, Jun Ma ${ }^{1}$ \\ ${ }^{1}$ Department of Radiation Oncology, Sun Yat-Sen University Cancer Center, State Key Laboratory of Oncology in Southern \\ China, Collaborative Innovation Center for Cancer Medicine, Guangzhou, People's Republic of China \\ ${ }^{2}$ Imaging Diagnosis and Interventional Center, Sun Yat-Sen University Cancer Center, State Key Laboratory of Oncology in \\ Southern China, Collaborative Innovation Center for Cancer Medicine, Guangzhou, People's Republic of China \\ ${ }^{3}$ Department of Medical Statistics and Epidemiology, School of Public Health, Sun Yat-Sen University, Guangzhou, People's \\ Republic of China
}

Correspondence to: Jun Ma, e-mail: majun2@mail.sysu.edu.cn

Keywords: nasopharyngeal carcinoma, primary tumor inflammation, gross tumor volume, prognosis, intensity-modulated radiation therapy

Received: October 28, 2015

Accepted: January 31, 2016

Published: February 25, 2016

\section{ABSTRACT}

Purpose: The objective of this study is to investigate the prognostic value of primary tumor inflammation (PTI) in nasopharyngeal carcinoma (NPC) in the era of intensity-modulated radiation therapy (IMRT).

Results: PTI was observed in 376/1708 (22.0\%) patients, and was present in the sphenoid sinus in $289 / 376(76.9 \%)$, in the nasal cavity in $27(7.2 \%)$, and in both places in 60 (15.9\%). The estimated 4-year local relapse-free survival (LRFS), diseasefree survival (DFS), overall survival (OS) and distant metastasis-free survival (DMFS) rates for PTI vs. non-PTI group were $89.2 \%$ vs. $96.1 \%(P<0.001), 73.4 \%$ vs. $85.1 \%$ $(P<0.001), 85.0 \%$ vs. $92.1 \%(P<0.001)$ and $83.6 \%$ vs. $91.4 \%(P<0.001)$, respectively. After adjustment for these known prognostic factors, PTI was confirmed as an independent prognostic factor for LRFS (HR 2.152, 95\% CI 1.318-3.516, $P=0.002$ ), DFS (HR 1.581, 95\% CI 1.204-2.077, $P=0.001$ ) and DMFS (HR 1.682, 95\% CI 1.177-2.402, $P=0.004$ ).

Conclusions: Primary tumor inflammation was identified as a strong prognostic factor for patients with NPC in the era of IMRT and should be considered when devising future treatment strategies aimed at improving survival in NPC patients.

Materials and Methods: Data on 1708 patients with nonmetastatic, histologicallyconfirmed NPC treated with IMRT between November 2009 and February 2012 at Sun Yat-Sen University Cancer Center were retrospectively reviewed. Patient survival between PTI and non-PTI groups were compared.

\section{INTRODUCTION}

Worldwide, there were an estimated 84,400 new cases of nasopharyngeal carcinoma (NPC) and 51,600 deaths in 2011 [1]. NPC is an uncommon cancer with a very unique geographical distribution, with an age-standardized incidence rate of 20-50 per 100,000 males in south China but only 0.5 per 100,000 in Caucasian populations in 2011 [1]. Due to anatomic constraints and high radiosensitivity, radiotherapy is the only curative treatment for NPC. Despite its known limitations, the TNM staging system remains the most important prognostic factor for NPC patients [2]. Many other factors had been reported to have prognostic value, including plasma Epstein-Barr virus (EBV) DNA [3-5], primary tumor volume [6, 7], pretreatment serum lactate dehydrogenase (LDH) levels [8] and apparent diffusion coefficient (ADC) [9].

Due to the special location and invasiveness of NPC [10], primary tumor inflammation (PTI) in gross tumor volume (GTV) is a commonly imaging feature in advanced 
T stage patients. Using magnetic resonance (MR) imaging, inflammation in GTV is seen as an area of high signal intensity on T2-weighted images, and as an area of low signal intensity on contrast material-enhanced T1-weighted images. The prognostic value of necrosis in cervical nodal, a subtype of inflammation in NPC patients, has been demonstrated [11], as has the prognostic role of necrosis in bladder cancer [12]. Therefore, it is reasonable to speculate that the inflammation in GTV may also have prognostic value in NPC.

As we know, the relationship between primary tumor inflammation in GTV and prognosis of NPC patients has not been studied. Hence, we conducted a large-scale retrospective study to evaluate the impact of PTI on the clinical features and survival outcomes of NPC patients based on MR imaging (MRI) results.

\section{RESULTS}

\section{Patient characteristics}

Of the 1708 patients, the male $(n=1273)$-to-female $(n=435)$ ratio was $2.9: 1$, the median age was 45 years (rang, 14-78 years). Patient characteristics are listed in Table 1. Of the 376 (22.0\%) patients with PTI, 168 (44.7\%) and $204(54.3 \%)$ were classified as $\mathrm{T}_{3}$ and $\mathrm{T}_{4}$, respectively $(P<0.001)$, while only $4(1.0 \%)$ were in disease stage $\mathrm{T}_{1-2}$. PTI was observed in the sphenoid sinus alone in 289 (76.9\%) patients, in the nasal cavity alone in $27(7.2 \%)$, and in both locations in $60(15.9 \%)$. PTI and non-PTI groups were similar in terms of drinking $(P=0.262)$. However, the PTI group had a higher percentage patients that were older $(P=0.009)$, male $(P=0.035)$, smokers $(P=0.002)$, classified as advanced T $(P<0.001)$ and $\mathrm{N}(P<0.001)$, and undergoing chemotherapy $(P<0.001)$.

\section{Patient failure patterns}

The median follow-up time for the entire cohort was 49.9 months (range, 1.3-76.4 months), and 263 (15.4\%) patients were lost to follow-up. Patterns of treatment failure and cause of death are summarized in Table 2. Up to the final follow-up, 39/376 (10.4\%) PTI patients and $52 / 1332$ (3.9\%) non-PTI patients experienced local failure $(P<0.001), 14 / 376$ (3.7\%) PTI patients and 55/1332 (4.1\%) non-PTI patients experienced regional failure $(P=0.724), 62 / 376(16.5 \%)$ PTI patients and $113 / 1332$ $(8.5 \%)$ non-PTI patients developed distant metastases $(P<0.001)$. Moreover, 59/376 (15.7\%) PTI patients and $108 / 1332(8.1 \%)$ non-PTI patients died, and the majority of deaths were attributed to NPC.

\section{Univariate and multivariate analysis}

The estimated 4-year local relapse-free survival (LRFS), disease-free survival (DFS), overall survival (OS) and distant metastasis-free survival (DMFS) rates for the whole cohort were $94.6 \%, 82.5 \%, 90.6 \%$ and $89.7 \%$, respectively. For PTI group vs. non-PTI group, they were $89.2 \%$ vs. $96.1 \%(P<0.001), 73.4 \%$ vs. $85.1 \%$ $(P<0.001), 85.0 \%$ vs. $92.1 \%(P<0.001)$ and $83.6 \%$ vs. 91.4\% $(P<0.001)$, respectively (Figure 1$)$.

The results of univariate analysis revealed that there was prognostic value in age greater than 50 years, pathology type, PTI, T and N classification, and overall stage. PTI, T classification and overall stage were associated with 4-year LRFS, DFS, OS and DMFS (Table 3). After adjusting for various prognostic factors, the outcomes of multivariate analysis showed that PTI was an independent prognostic factor for LRFS (HR 2.152, 95\% CI 1.318-3.516, $P=0.002)$, DFS (HR 1.581, 95\% CI 1.204-2.077, $P=0.001)$ and DMFS (HR 1.682, 95\% CI 1.177-2.402, $P=0.004)$ (Table 4).

\section{Subgroup analysis}

Due to the unbalanced distribution of PTI in T classification, subgroup analysis according to $\mathrm{T}$ and $\mathrm{N}$ classification and overall stage was performed to further investigate the prognostic value of PTI (Table 5). The survival outcomes for PTI patients classified as T3 were substantially poorer than those T3 patients in the no-PTI group $(P=0.001$ for LRFS, $P=0.005$ for DFS, $P=0.041$ for DMFS), and were similar to those of patients classified asT4 in the no-PTI group (90.6\% vs. 90.9 for LRFS, $76.6 \%$ vs. $77.8 \%$ for DFS, $86 \%$ vs. $86.7 \%$ for DMFS). Additionally, for patients with N1 disease, the presence of PTI markedly affected prognosis $(P<0.05$ for all rates). However, the presence of PTI did not substantially affect the outcomes of patients with T4 and N2-3 disease $(P>0.05$ for all rates).

\section{DISCUSSION}

To the best of our knowledge, this is the first largescale study to investigate the prognostic value of PTI in NPC. In the present study, we observed a relatively high incidence $(22.0 \%)$ of PTI, especially in patients with advanced $T$ stage. The results of this study revealed that PTI was an independent prognostic factor with regard to 4-year LRFS, DFS and DMFS for patients with nonmetastatic NPC in the era of IMRT. However, no significant difference was found in 4-year OS for these two groups.

Due to the anatomical specificity and invasiveness, inflammation is common in the sphenoid sinus and nasal cavity in advanced T stage NPC and often combines with necrosis and bacterial infections, which results in local hypoxia and radioresistance. This was also observed in head and neck cancers [13-16]. Moreover, numerous previous studies showed that hypoxia is also an adverse prognostic factor in many malignant cancers like lung, breast, uterine cervix, rectum, brain, soft tissue and renal cell [17-23]. This mechanism may also explain the unfavorable prognostic value of cervical nodal necrosis in NPC [11]. 
Table 1: Baseline characteristics of the 1708 patients included in this study

\begin{tabular}{|c|c|c|c|c|}
\hline \multirow{2}{*}{ Characteristics } & PTI group & Non-PTI group & \multirow{2}{*}{ Total } & \multirow{2}{*}{$P^{a}$} \\
\hline & No. (\%) & No. $(\%)$ & & \\
\hline Total & 376 & 1332 & 1708 & \\
\hline Age (years) & & & & 0.009 \\
\hline$<50$ & $236(62.8)$ & $931(69.9)$ & 1167 & \\
\hline$\geq 50$ & $140(37.2)$ & $401(30.1)$ & 541 & \\
\hline$\overline{\text { Sex }}$ & & & & 0.035 \\
\hline Male & $296(78.7)$ & $977(73.3)$ & 1273 & \\
\hline Female & $80(21.3)$ & $355(26.7)$ & 435 & \\
\hline WHO pathology & & & & 0.001 \\
\hline Type I & $6(1.6)$ & $3(0.2)$ & 9 & \\
\hline Type II/III & $370(98.4)$ & $1329(99.8)$ & 1699 & \\
\hline Smoking & & & & 0.002 \\
\hline Yes & $163(43.4)$ & $460(34.5)$ & 623 & \\
\hline No & $213(56.6)$ & $872(65.5)$ & 1085 & \\
\hline Drinking & & & & 0.262 \\
\hline Yes & $53(14.1)$ & 159 (11.9) & 212 & \\
\hline No & $323(85.9)$ & $1173(88.1)$ & 1496 & \\
\hline $\mathrm{T}_{\text {classification }}{ }^{\mathrm{b}}$ & & & & $<0.001$ \\
\hline $\mathrm{T} 1$ & $2(0.5)$ & $307(23.1)$ & 309 & \\
\hline $\mathrm{T} 2$ & $2(0.5)$ & $258(19.4)$ & 260 & \\
\hline $\mathrm{T} 3$ & $168(44.7)$ & $648(48.6)$ & 816 & \\
\hline $\mathrm{T} 4$ & $204(54.3)$ & $119(8.9)$ & 323 & \\
\hline $\mathrm{N}$ classification ${ }^{\mathrm{b}}$ & & & & $<0.001$ \\
\hline N0 & $33(8.8)$ & $253(19.0)$ & 286 & \\
\hline N1 & $242(64.4)$ & $764(57.3)$ & 1006 & \\
\hline $\mathrm{N} 2$ & $76(20.2)$ & $190(14.3)$ & 266 & \\
\hline N3 & $25(6.6)$ & $125(9.4)$ & 150 & \\
\hline Overall stage $^{\mathrm{b}}$ & & & & $<0.001$ \\
\hline I & $0(0)$ & $92(6.9)$ & 92 & \\
\hline II & $2(0.5)$ & $353(26.5)$ & 355 & \\
\hline III & $158(42.0)$ & $657(49.3)$ & 815 & \\
\hline IVA-IVB & $216(57.5)$ & $230(17.3)$ & 446 & \\
\hline Chemotherapy & & & & $<0.001$ \\
\hline Yes & $366(97.3)$ & $1113(83.6)$ & 1479 & \\
\hline No & $10(2.7)$ & $219(16.4)$ & 229 & \\
\hline
\end{tabular}

Abbreviations: $\mathrm{PTI}=$ primary tumor inflammation; $\mathrm{WHO}=$ World Health Organization .

${ }^{a} P$ values were calculated using chi-square or Fisher exact test as indicated.

${ }^{\mathrm{b}}$ According to the 7 th edition of the AJCC/UICC staging system. 
Table 2: Treatment failure patterns and cause of death

\begin{tabular}{|l|c|c|c|}
\hline \multirow{2}{*}{\multicolumn{1}{c}{ Failure patterns }} & No. $(\%)$ & \multicolumn{2}{c}{ Non-PTI group } \\
\cline { 2 - 3 } \multicolumn{1}{c|}{$\boldsymbol{P}^{\mathbf{2}}$} & $27(2.0)$ & $<0.001$ \\
\hline Local only & $29(7.7)$ & $11(0.8)$ & 0.911 \\
\hline Local + regional & $2(0.5)$ & $9(0.7)$ & 0.172 \\
\hline Local + distant & $6(1.6)$ & $5(0.4)$ & 1.000 \\
\hline Local + regional + distant & $2(0.5)$ & $32(2.4)$ & 0.206 \\
\hline Regional only & $4(1.1)$ & $7(0.5)$ & 0.024 \\
\hline Regional + distant & $6(1.6)$ & $91(6.8)$ & $<0.001$ \\
\hline Distant only & $48(12.8)$ & $92(6.9)$ & $<0.001$ \\
\hline Total locoregional & $49(13.0)$ & $113(8.5)$ & $<0.001$ \\
\hline Total distant & $62(16.5)$ & 183 & \\
\hline Total & 97 & & 0.406 \\
\hline Cause of death & & $90(83.3)$ & \\
\hline Cancer & $52(88.1)$ & $18(16.7)$ & \\
\hline Non-cancer & $7(11.9)$ & 108 & \\
\hline Total & 59 & & \\
\hline
\end{tabular}

Abbreviations: PTI = primary tumor inflammation.

${ }^{a} P$ values were calculated using chi-square or Fisher exact test as indicated.
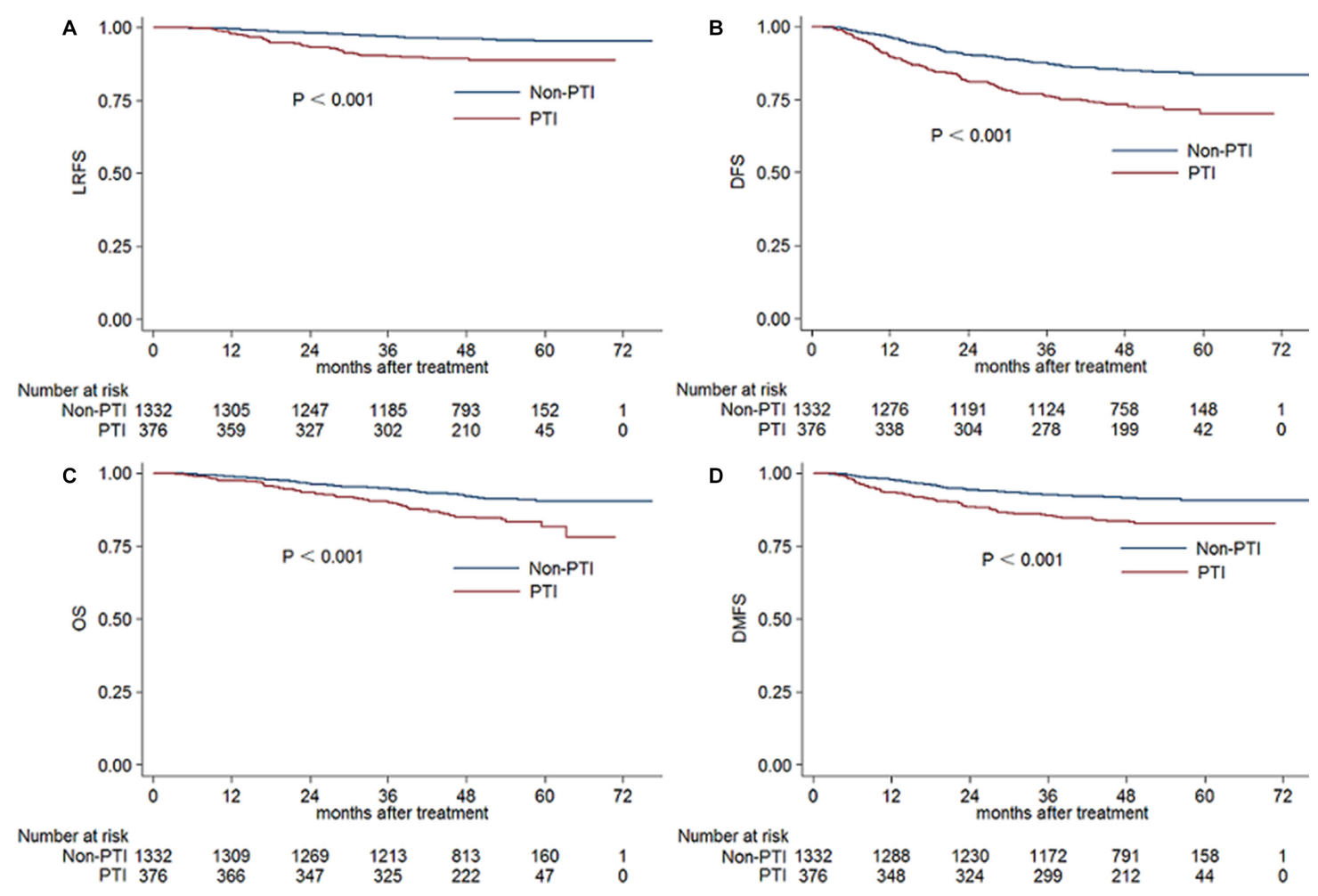

Figure 1: Kaplan-Meier LRFS. (A), DFS (B), OS (C) and DMFS (D) curves for PTI and non-PTI patients. Abbreviations: LRFS = local relapse-free survival; DFS = disease-free survival; OS = overall survival; DMFS = distant metastasis-free survival; PTI = primary tumor inflammation. 
Table 3: Univariate analysis of variables associated with prognostic outcomes

\begin{tabular}{|c|c|c|c|c|c|c|c|c|c|}
\hline Characteristics & Patients & $\begin{array}{l}\text { 4-year } \\
\text { LRFS }\end{array}$ & $P^{\mathbf{a}}$ & $\begin{array}{c}\text { 4-year } \\
\text { DFS }\end{array}$ & $P^{\mathbf{a}}$ & $\begin{array}{c}\text { 4-year } \\
\text { OS }\end{array}$ & $P^{\mathrm{a}}$ & $\begin{array}{l}\text { 4-year } \\
\text { DMFS }\end{array}$ & $P^{\mathbf{a}}$ \\
\hline & No. $(\%)$ & $(\%)$ & & $(\%)$ & & $(\%)$ & & $(\%)$ & \\
\hline Age (years) & & & 0.329 & & 0.002 & & $<0.001$ & & 0.296 \\
\hline$<50$ & $1167(68.3)$ & 94.9 & & 84.4 & & 92.6 & & 90.2 & \\
\hline$\geq 50$ & $541(31.7)$ & 94.0 & & 78.3 & & 86.0 & & 88.5 & \\
\hline Sex & & & 0.105 & & 0.876 & & 0.143 & & 0.276 \\
\hline Male & $1273(74.5)$ & 95.1 & & 82.4 & & 89.9 & & 89.2 & \\
\hline Female & $435(25.5)$ & 93.2 & & 82.6 & & 92.5 & & 91.2 & \\
\hline WHO pathology & & & $<0.001$ & & 0.011 & & 0.008 & & 0.353 \\
\hline Type I & $9(0.5)$ & 66.7 & & 55.6 & & 66.7 & & 100 & \\
\hline Type II/III & $1699(99.5)$ & 94.8 & & 82.6 & & 90.7 & & 89.6 & \\
\hline Primary tumor & & & $<0.001$ & & $<0.001$ & & $<0.001$ & & $<0.001$ \\
\hline PTI & $376(22.0)$ & 89.2 & & 73.4 & & 85.0 & & 83.6 & \\
\hline Non-PTI & $1332(78.0)$ & 96.1 & & 85.1 & & 92.1 & & 91.4 & \\
\hline Smoking & & & 0.603 & & 0.017 & & 0.005 & & 0.102 \\
\hline Yes & $623(36.5)$ & 94.3 & & 79.6 & & 88.1 & & 88.0 & \\
\hline No & $1085(63.5)$ & 94.8 & & 84.2 & & 92.0 & & 90.6 & \\
\hline Drinking & & & 0.176 & & 0.287 & & 0.174 & & 0.687 \\
\hline Yes & $212(12.4)$ & 96.4 & & 79.1 & & 88.5 & & 88.5 & \\
\hline No & $1496(87.6)$ & 94.4 & & 83.0 & & 90.9 & & 89.8 & \\
\hline $\mathrm{T}$ classification & & & $<0.001$ & & $<0.001$ & & $<0.001$ & & $<0.001$ \\
\hline $\mathrm{T} 1$ & $309(18.1)$ & 97.2 & & 88.7 & & 96.9 & & 93.6 & \\
\hline $\mathrm{T} 2$ & $260(15.2)$ & 95.1 & & 84.7 & & 93.6 & & 91.8 & \\
\hline $\mathrm{T} 3$ & $816(47.8)$ & 95.6 & & 83.1 & & 90.5 & & 90.0 & \\
\hline $\mathrm{T} 4$ & $323(18.9)$ & 89.0 & & 73.3 & & 82.4 & & 83.5 & \\
\hline $\mathrm{N}$ classification & & & 0.552 & & $<0.001$ & & $<0.001$ & & $<0.001$ \\
\hline N0 & $286(16.7)$ & 95.6 & & 92.1 & & 95.9 & & 95.8 & \\
\hline N1 & $1006(58.9)$ & 94.5 & & 84.2 & & 92.8 & & 92.0 & \\
\hline $\mathrm{N} 2$ & $266(15.6)$ & 93.3 & & 75.5 & & 85.3 & & 83.7 & \\
\hline N3 & $150(8.8)$ & 96.0 & & 64.3 & & 74.2 & & 72.2 & \\
\hline Overall stage & & & 0.002 & & $<0.001$ & & $<0.001$ & & $<0.001$ \\
\hline I & $92(5.4)$ & 98.7 & & 97.6 & & 98.9 & & 98.9 & \\
\hline II & $355(20.8)$ & 95.7 & & 88.6 & & 94.6 & & 94.6 & \\
\hline III & $815(47.7)$ & 95.5 & & 84.4 & & 91.4 & & 91.4 & \\
\hline IV & $446(26.1)$ & 91.0 & & 70.9 & & 80.6 & & 80.6 & \\
\hline
\end{tabular}

Abbreviations: PTI = primary tumor inflammation; LRFS = local relapse-free survival; DFS = disease-free survival; OS = overall survival; DMFS = distant metastases-free survival; WHO = world health organization.

${ }^{a} P$ values were calculated using the log-rank test. 
Table 4: Multivariate analysis of prognostic factors correlated with clinical outcomes

\begin{tabular}{|l|l|c|c|c|}
\hline \multicolumn{1}{c}{ Endpoint } & \multicolumn{1}{c}{ Variable } & H & HR & 95\% CI for HR \\
\hline LRFS & PTI & 0.002 & 0.152 & $1.318-3.516$ \\
\hline & Pathology & 0.002 & 0.163 & $0.051-0.552$ \\
\hline DFS & Age & 0.014 & 1.338 & $1.061-1.686$ \\
\hline & PTI & 0.001 & 1.581 & $1.204-2.077$ \\
\hline & T classification & 0.019 & 1.185 & $1.029-1.366$ \\
\hline & N classification & $<0.001$ & 1.656 & $1.455-1.884$ \\
\hline OS & Age & $<0.001$ & 1.778 & $1.309-2.414$ \\
\hline & T classification & $<0.001$ & 1.759 & $1.455-2.125$ \\
\hline & N classification & $<0.001$ & 1.943 & $1.638-2.304$ \\
\hline DMFS & PTI & 0.004 & 1.682 & $1.177-2.402$ \\
\hline & N classification & $<0.001$ & 2.013 & $1.705-2.377$ \\
\hline
\end{tabular}

Abbreviations: PTI $=$ primary tumor inflammation; LRFS $=$ local relapse-free survival; DFS $=$ disease-free survival; $\mathrm{OS}=$ overall survival; DMFS = distant metastases-free survival; $\mathrm{HR}=$ hazard ratio; $\mathrm{CI}=$ confidence interval.

${ }^{a}$ Multivariate $P$-values were calculated using an adjusted Cox proportional-hazards model with backward elimination and the following parameters: age ( $\geq 50$ y vs. $<50$ y), gender (male or female), pathological type (type I or type II/III), PTI (yes or no), smoking (yes or no), drinking (yes or no), T classification, $\mathrm{N}$ classification, undergoing chemotherapy (yes or No).

In our cohort, patients with PTI tended to be older than patients without PTI, indicating that age may adversely contribute to primary tumor inflammation. One reasonable explanation is that older patients are likely to be in worse physical condition. PTI patients were also at a more advanced clinical stage on average, and tumor volume was generally larger. A greater number of PTI patients were thus undergoing chemotherapy. The significant prognostic difference showed by univariate analysis between these two groups should originate from unbalanced TNM staging and other prognostic factors. Multivariate analysis revealed PTI was an prognostic factor for LRFS, DFS and DMFS but not for OS, which indicated that the follow-up time was insufficient, and should be longer in any similar studies in future.

Subgroup analysis revealed a difference in the prognosis of PTI patients with T3 or N1 disease. Due to the extremely low incidence of PTI, no clinical prognostic value in T1-2 or N0 patients was observed. Moreover, patients with T4 or N2-3 are at a higher risk of distant metastases, and the prognostic impact of PTI may be masked by other important prognostic factors. The survival outcomes of PTI patients with T3 disease were similar to those with T4 classification. Hence, we proposed that T3 stage should be divided intoT3a and T3b stage based on PTI. More intensive chemotherapy regimen may be needed for patients with PTI compared with patients without PTI .
Another reason for unfavorable prognosis of PTI patients may be due to the difficulty in determining an accurate GTV using IMRT. Inflammation in the sphenoid sinus and nasal cavity often mixed with tumor and made it difficult to establish the tumor margin. Hence, the tumor target would easily be left out. Therefore, a thorough pretreatment assessment of PTI patients undergoing IMRT should be performed, and accurate delineation of GTV should be warranted to reduce local recurrence.

The findings of our current study suggest that clinicians should pay particular attention to NPC patients with PTI in order to accurately delineate the tumor target. Induction chemotherapy could be applied to shrink the tumor bulk and minimize inflammation to assist identification of the tumor margin. Additionally, a better dose coverage and reduced toxicity from radiotherapy could be achieved after induction chemotherapy. Additionally, proton and heavy particle therapy could result in better prognosis [24].

The main limitation of our study was that the judgement of PTI was only based on MRI. However, this could not be avoided because pathological results from surgical resection are not available due to the special location of NPC. The retrospective nature and short followup time are also limitations that should be addressed in future studies, and additional clinical experiments are needed to establish the prognostic value of PTI. 
Table 5: Subgroup analysis of T, N and overall stage of PTI and non-PTI groups

\begin{tabular}{|c|c|c|c|c|c|c|c|c|c|c|c|c|}
\hline \multirow[b]{2}{*}{ Stage } & \multicolumn{3}{|c|}{ 4-year LRFS } & \multicolumn{3}{|c|}{ 4-year DFS } & \multicolumn{3}{|c|}{ 4-year OS } & \multicolumn{3}{|c|}{ 4-year DMFS } \\
\hline & $\begin{array}{l}\text { PTI } \\
(\%)\end{array}$ & $\begin{array}{c}\text { Non- } \\
\text { PTI } \\
(\%)\end{array}$ & $P^{a}$ & $\begin{array}{l}\text { PTI } \\
(\%)\end{array}$ & $\begin{array}{c}\text { Non- } \\
\text { PTI } \\
(\%)\end{array}$ & $P^{a}$ & $\begin{array}{l}\text { PTI } \\
(\%)\end{array}$ & $\begin{array}{c}\text { Non- } \\
\text { PTI } \\
(\%)\end{array}$ & $P^{a}$ & $\begin{array}{l}\text { PTI } \\
(\%)\end{array}$ & $\begin{array}{c}\text { Non- } \\
\text { PTI } \\
(\%)\end{array}$ & $P^{\mathbf{a}}$ \\
\hline \multicolumn{13}{|l|}{$\mathrm{T}$} \\
\hline $\mathrm{T} 1$ & 100 & 97.2 & 0.787 & 50 & 88.9 & 0.059 & 100 & 96.9 & 0.801 & 50 & 93.9 & 0.006 \\
\hline $\mathrm{T} 2$ & 100 & 95.1 & 0.740 & 100 & 84.6 & 0.558 & 100 & 93.6 & 0.716 & 100 & 91.7 & 0.678 \\
\hline $\mathrm{T} 3$ & 90.6 & 96.9 & 0.001 & 76.6 & 84.7 & 0.005 & 89 & 90.9 & 0.181 & 86 & 91 & 0.041 \\
\hline $\mathrm{T} 4$ & 87.9 & 90.9 & 0.331 & 70.7 & 77.8 & 0.138 & 81.5 & 84 & 0.954 & 81.7 & 86.7 & 0.192 \\
\hline \multicolumn{13}{|l|}{$\mathrm{N}$} \\
\hline N0 & 90.9 & 96.2 & 0.153 & 81.8 & 93.5 & 0.022 & 89.8 & 96.8 & 0.018 & 90.9 & 96.4 & 0.129 \\
\hline N1 & 88.8 & 96.2 & $<0.001$ & 74.9 & 87.2 & $<0.001$ & 86.7 & 94.8 & $<0.001$ & 86.8 & 93.7 & 0.001 \\
\hline $\mathrm{N} 2$ & 89.7 & 94.7 & 0.144 & 70.6 & 77.5 & 0.160 & 85 & 85.4 & 0.655 & 78.3 & 85.8 & 0.07 \\
\hline N3 & 88.2 & 97.3 & 0.136 & 55.2 & 66.2 & 0.268 & 61 & 76.6 & 0.173 & 59.5 & 74.9 & 0.073 \\
\hline \multicolumn{13}{|c|}{ Overall } \\
\hline I & - & 98.7 & - & - & 97.6 & - & - & 100 & - & - & 98.9 & - \\
\hline II & 100 & 95.6 & 0.749 & 100 & 88.5 & 0.612 & 100 & 97.9 & 0.837 & 100 & 94.6 & 0.739 \\
\hline III & 90.8 & 96.6 & 0.002 & 77.7 & 86.1 & 0.003 & 90.3 & 91.9 & 0.137 & 87.1 & 92.4 & 0.024 \\
\hline IV & 87.9 & 94.1 & 0.021 & 69.9 & 71.9 & 0.539 & 80.9 & 80.8 & 0.494 & 80.8 & 80.5 & 0.89 \\
\hline
\end{tabular}

Abbreviations: PTI = primary tumor inflammation; LRFS = local relapse-free survival; DFS = disease-free survival; OS = overall survival; DMFS = distant metastases-free survival.

${ }^{a} P$ values were calculated using the log-rank test.

-No patients with stage I disease had PTI.

\section{CONCLUSIONS}

In summary, this study confirmed that PTI was an independent prognostic factor for LRFS, DFS and DMFS for patients with NPC in the era of IMRT. It is advised that T3 stage should be divided into T3a and T3b stage based on the presence of PTI, and different intense treatment protocols should be considered for patients with PTI. Further prospective clinical study should be warranted to confirm the results of this current study.

\section{MATERIALS AND METHODS}

\section{Patients}

We retrospectively analyzed 1811 patients with newly diagnosed NPC that showed no evidence of distant metastasis, who were treated between November 2009 and
February 2012 at Sun Yat-Sen University Cancer Center. Pre-treatment MR images of the nasopharynx and cervical region were thoroughly analyzed. Of the entire cohort, 103 (5.7\%) patients without MRI results were excluded, which left $1708(94.3 \%)$ patients for further investigation. This study was approved by the Research Ethics Committee of Sun Yat-Sen University Cancer Center, and informed consent was obtained from all patients.

\section{Clinical staging}

The routine staging process included a complete history and clinical examination of the head and neck region, direct fibre-optic nasopharyngoscopy, MRI scans of the skull base, neck and chest radiography, a whole-body bone scan, and abdominal sonography. Positron emission tomography (PET)-CT scans were also performed if clinical indicated. All patients received a dental evaluation before radiotherapy. 
All patients were restaged according to the 7 th edition of the International Union against Cancer/American Joint Committee on Cancer (UICC/AJCC) system [25]. All MRI materials and clinical records were reviewed to minimize heterogeneity in restaging. Two radiologists employed at our hospital separately evaluated all of the scans and disagreements were resolved by consensus.

\section{Diagnostic criteria for PTI}

All patients underwent MRI scans using a 3 Tesla system (Trio Tim; Siemens, Erlangen Germany). Only patients with inflammation surrounding the tumor were included in this study, and patients with sinusitis not in the immediate vicinity of the tumor were excluded. Diagnostic criteria for primary tumor inflammation in MRI include an area of high signal intensity on T2weighted images, and an area of low signal intensity on contrast material-enhanced T1-weighted images, which is similar to the diagnostic criteria for lymph node necrosis (Figure 2) [26, 27].

\section{Clinical treatment}

\section{Radiotherapy}

All patients received intensity-modulated radiation therapy (IMRT) at Sun Yat-Sen University Cancer Center. Immobilization was carried out using a custom-made head-to-neck-thermoplastic cast with the patient's neck
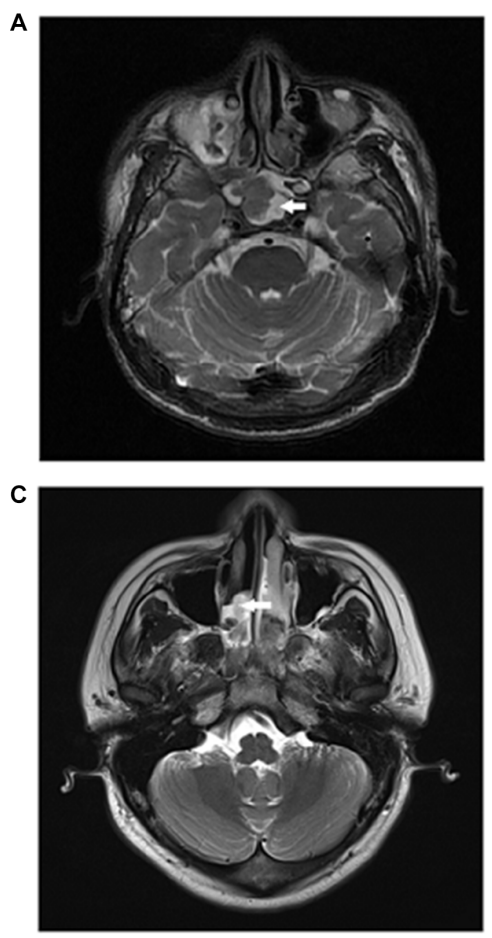

resting on a support. A high-resolution planning computed tomography scan with contrast was taken from the vertex to $2 \mathrm{~cm}$ below the sternoclavicular joint at a slice thickness of $3 \mathrm{~mm}$. Target volumes were delineated slice-by-slice on treatment planning $\mathrm{CT}$ scans using an individualized delineation protocol that complies with International Commission on Radiation Units and Measurements report numbers 50 and 62. The prescribed doses were 66-72 Gy at 2.12-2.43 Gy/fraction to the planning target volume (PTV) of the primary gross tumour volume (GTVnx), 64-70 Gy to the PTV of the GTV of the involved lymph nodes (GTVnd), 60-63 Gy to the PTV of the high-risk clinical target volume (CTV1), and 54-56 Gy to the PTV of the low-risk clinical target volume (CTV2). All targets were treated simultaneously using the simultaneous integrated boost technique.

\section{Chemotherapy}

According to our institutional guidelines, prior to commencing treatment we recommended radiotherapy alone for stage I disease, concurrent chemoradiotherapy (CCRT) for stage II disease, and CCRT +/- neoadjuvant/ adjuvant chemotherapy for stage III to IVA-B disease. Neoadjuvant or adjuvant chemotherapy consisted of cisplatin with 5-fluorouracil, cisplatin with taxoids or cisplatin with 5-fluorouracil and toxoids, every three weeks for two or three cycles. Concurrent chemotherapy consisted of cisplatin given weekly or on weeks 1, 4 and 7 of radiotherapy.
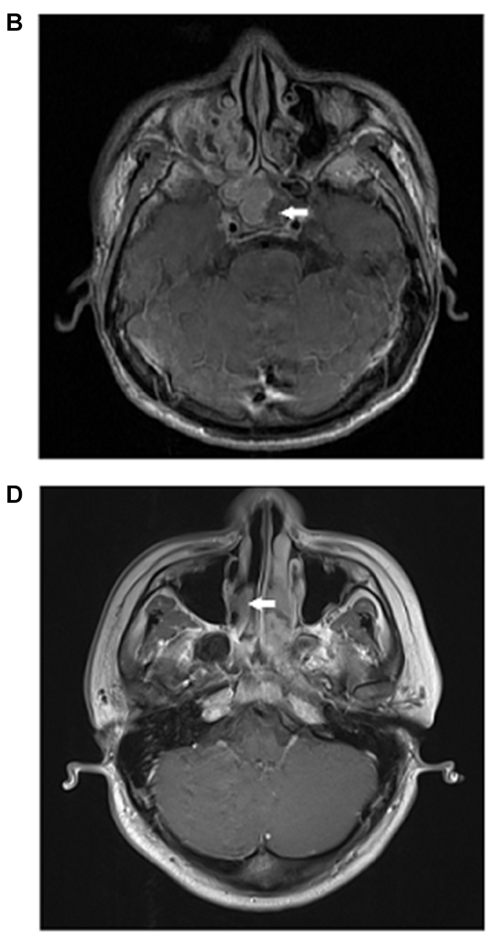

Figure 2: Primary tumor inflammation in two NPC patients. (A) Axial T2-weighted and (B) contrast-enhanced T1-weighted MR images of a 41-year-old man show primary tumor inflammation in the sphenoid sinus; (C) Axial T2-weighted and (D) contrast-enhanced T1-weighted MR images of a 29-year-old man show primary tumor inflammation in the nasal cavity. 


\section{Follow-up and statistical analysis}

Patients were followed-up from the first day of therapy to the day of last examination or death, and were examined at least every three months during the first two years, with follow-up examinations every six months thereafter until death. The end points (time to the first defining event) included LRFS, DFS, OS, and DMFS. LRFS was chosen as the first endpoint in this study.

Chi-square or Fisher exact tests were used to compare the categorical characteristics and treatment failure patterns between PTI and non-PTI groups, and subgroups were analyzed according to $\mathrm{T}$ classification, $\mathrm{N}$ classification, and overall stages. Life-table estimation was performed using the Kaplan-Meier method and differences were compared using the log-rank test. The multivariate Cox proportional hazards model was used to estimate the hazard ratio (HR) and to calculate $95 \%$ confidence intervals (CI). Variables in the model included age, gender, pathology, T classification, $\mathrm{N}$ classification, chemotherapy, and PTI. All statistical tests were two-sided, and $P<0.05$ was considered statistically significant. The STATA statistical package (STATA 12; StataCorp LP, College Station, TX, USA) was used for all analyses.

\section{GRANT SUPPORT}

This work was supported by grants from the Key Laboratory Construction Project of Guangzhou City, China (No.121800085), the Health \& Medical Collaborative Innovation Project of Guangzhou City, China (201400000001), the Sun Yat-Sen University Clinical Research 5010 Program (No. 2012011), the Science and Technology Project of Guangzhou City, China (No. 14570006), and the Planned Science and Technology Project of Guangdong Province (No. 2013B020400004).

\section{CONFLICTS OF INTEREST}

The authors declare no conflicts of interest.

\section{REFERENCES}

1. Jemal A, Bray F, Center MM, Ferlay J, Ward E, Forman D. Global cancer statistics. CA Cancer J Clin. 2011; 61:69-90.

2. Xu L, Pan J, Wu J, Pan C, Zhang Y, Lin S, Yang L, Chen C, Zhang C, Zheng W, Lin S, Ni X, Kong FM. Factors associated with overall survival in 1706 patients with nasopharyngeal carcinoma: significance of intensive neoadjuvant chemotherapy and radiation break. Radiother Oncol. 2010; 96:94-9.

3. Lin JC, Wang WY, Chen KY, Wei YH, Liang WM, Jan JS, Jiang RS. Quantification of plasma Epstein-Barr virus DNA in patients with advanced nasopharyngeal carcinoma. N Engl J Med. 2004; 350:2461-70.
4. Lin JC, Wang WY, Liang WM, Chou HY, Jan JS, Jiang RS, Wang JY, Twu CW, Liang KL, Chao J, Shen WC. Long-term prognostic effects of plasma epstein-barr virus DNA by minor groove binder-probe real-time quantitative PCR on nasopharyngeal carcinoma patients receiving concurrent chemoradiotherapy. Int J Radiat Oncol Biol Phys. 2007; 68:1342-8.

5. Lo YM, Chan LY, Chan AT, Leung SF, Lo KW, Zhang J, Lee JC, Hjelm NM, Johnson PJ, Huang DP. Quantitative and temporal correlation between circulating cell-free EpsteinBarr virus DNA and tumor recurrence in nasopharyngeal carcinoma. Cancer Res. 1999; 59:5452-5.

6. Guo R, Sun Y, Yu XL, Yin WJ, Li WF, Chen YY, Mao YP, Liu LZ, Li L, Lin AH, Ma J. Is primary tumor volume still a prognostic factor in intensity modulated radiation therapy for nasopharyngeal carcinoma? Radiother Oncol. 2012; 104:294-9.

7. Tian YM, Xiao WW, Bai L, Liu XW, Zhao C, Lu TX, Han F. Impact of primary tumor volume and location on the prognosis of patients with locally recurrent nasopharyngeal carcinoma. Chin J Cancer. 2015; 34:247-53.

8. Wan XB, Wei L, Li H, Dong M, Lin Q, Ma XK, Huang PY, Wen JY, Li X, Chen J, Ruan DY, Lin ZX, Chen ZH, et al. High pretreatment serum lactate dehydrogenase level correlates with disease relapse and predicts an inferior outcome in locally advanced nasopharyngeal carcinoma. Eur J Cancer. 2013; 49:2356-64.

9. Zhang Y, Liu X, Zhang Y, Li WF, Chen L, Mao YP, Shen JX, Zhang F, Peng H, Liu Q, Sun Y, Ma J. Prognostic value of the primary lesion apparent diffusion coefficient (ADC) in nasopharyngeal carcinoma: a retrospective study of 541 cases. Sci Rep. 2015; 5:12242.

10. Chua ML, Wee JT, Hui EP, Chan AT. Nasopharyngeal carcinoma. Lancet. 2015.

11. Lan M, Huang Y, Chen CY, Han F, Wu SX, Tian L, Zheng L, Lu TX. Prognostic Value of Cervical Nodal Necrosis in Nasopharyngeal Carcinoma: Analysis of 1800 Patients with Positive Cervical Nodal Metastasis at MR Imaging. Radiology. 2015; 276:619.

12. Eustace A, Irlam JJ, Taylor J, Denley H, Agrawal S, Choudhury A, Ryder D, Ord JJ, Harris AL, Rojas AM, Hoskin PJ, West CM. Necrosis predicts benefit from hypoxiamodifying therapy in patients with high risk bladder cancer enrolled in a phase III randomised trial. Radiother Oncol. 2013; 108:40-7.

13. Biau J, Chautard E, Miroir J, Lapeyre M. Radioresistance parameters in head and neck cancers and methods to radiosensitize. [Article in French]. Cancer Radiother. 2015; 19:337-46.

14. Brizel DM, Sibley GS, Prosnitz LR, Scher RL, Dewhirst MW. Tumor hypoxia adversely affects the prognosis of carcinoma of the head and neck. Int J Radiat Oncol Biol Phys. 1997; 38:285-9.

15. Overgaard J. Hypoxic modification of radiotherapy in squamous cell carcinoma of the head and neck - a systematic review and meta-analysis. Radiother Oncol. 2011; 100:22-32. 
16. Overgaard J, Horsman MR. Modification of HypoxiaInduced Radioresistance in Tumors by the Use of Oxygen and Sensitizers. Semin Radiat Oncol. 1996; 6:10-21.

17. Hockel M, Schlenger K, Hockel S, Aral B, Schaffer U, Vaupel P. Tumor hypoxia in pelvic recurrences of cervical cancer. Int J Cancer. 1998; 79:365-9.

18. Vaupel P, Briest S, Hockel M. Hypoxia in breast cancer: pathogenesis, characterization and biological/therapeutic implications. Wien Med Wochenschr. 2002; 152:334-42.

19. Vaupel P, Hockel M, Mayer A. Detection and characterization of tumor hypoxia using pO2 histography. Antioxid Redox Signal. 2007; 9:1221-35.

20. Vaupel P, Mayer A. Hypoxia and anemia: effects on tumor biology and treatment resistance. Transfus Clin Biol. 2005; 12:5-10

21. Vaupel P, Mayer A, Briest S, Hockel M. Hypoxia in breast cancer: role of blood flow, oxygen diffusion distances, and anemia in the development of oxygen depletion. Adv Exp Med Biol. 2005; 566:333-42.

22. Vaupel P, Mayer A, Hockel M. Tumor hypoxia and malignant progression. Methods Enzymol. 2004; 381:335-54.
23. Vaupel P, Thews O, Hoeckel M. Treatment resistance of solid tumors: role of hypoxia and anemia. Med Oncol. 2001; $18: 243-59$.

24. Lewis GD, Holliday EB, Kocak-Uzel E, Hernandez M, Garden AS, Rosenthal DI, Frank SJ. Intensity-modulated proton therapy for nasopharyngeal carcinoma: Decreased radiation dose to normal structures and encouraging clinical outcomes. Head Neck. 2015.

25. Edge SB, Compton CC. The American Joint Committee on Cancer: the 7th edition of the AJCC cancer staging manual and the future of TNM. Ann Surg Oncol, 2010. 17:1471-4.

26. Chong VF, Fan YF, Khoo JB. MRI features of cervical nodal necrosis in metastatic disease. Clin Radiol. 1996; 51:103-9.

27. King AD, Tse GM, Ahuja AT, Yuen EH, Vlantis AC, To EW, Van Hasselt AC. Necrosis in metastatic neck nodes: diagnostic accuracy of CT, MR imaging, and US. Radiology. 2004; 230:720-6. 